\title{
LIE ALGEBRAS OF VECTOR FIELDS AND CODIMENSION ONE FOLIATIONS
}

\author{
TOMASZ RYBICKI
}

\begin{abstract}
The main result is a Pursell-Shanks type theorem for codimension one foliations. This theorem can be viewed as a partial solution of a hypothetical general version of the theorem of Pursell-Shanks. Several propositions and lemmas on foliations are contained in the proof.
\end{abstract}

\section{Introduction}

The Lie algebra $\mathcal{X}(M)$ consisting of all vector fields on a smooth manifold $M$ gives an important example of an infinite dimensional Lie algebra. A fundamental theorem proved by L.E. Pursell and M.E. Shanks in [10] states that the Lie algebra structure of $\mathcal{X}(M)$ completely determines the underiying topological and smooth structure of $M$. A whole series of papers followed, e.g. [1], [6], [9]. Our object of interest is the Lie algebra of a codimension one foliation.

Let $\left(M_{1}, F_{1}\right)$ and $\left(M_{2}, F_{2}\right)$ be smooth, second countable and smoothly foliated manifolds. By $\mathcal{Y}\left(M_{i}, F_{i}\right), i=1,2$, we denote the Lie algebra of all leaf prescrving vector fields on $M_{i}$. I. Amemiya in [1] proved a theorem which can be formulated as follows.

Theorem 1.1. If there exists a Lie algebra isomorphism $\Phi$ of $\mathcal{Y}\left(M_{1}, F_{1}\right)$ onto $\mathcal{Y}\left(M_{2}, F_{2}\right)$ then there exists a foliation preserving diffeomorphism $\varphi$ of $M_{1}$ anto $M_{2}$ such that $\varphi_{*}=\Phi$.

This note is devoted mainly to a generalization of Theorem 1.1. For any foliated manifold $(M, F)$ the symbol $\mathcal{X}(M, F)$ stands for the Lie algebra of all foliated vector fields i.e. vector fields with a flow transforming each leaf of $F$ onto a leaf of $F$. We recall that $X$ is a foliated vector field if $[X, Y] \in \mathcal{Y}(M, F)$ for any $Y \in \mathcal{Y}(M, F)$. Thus $\mathcal{Y}(M, F)$ is an ideal of $\mathcal{X}(M, F)$.

Now our result is the following 
Theorem 1.2. Let $\left(M_{1}, F_{1}\right)$ and $\left(M_{2}, F_{2}\right)$ be one codimensioal smooth nontrivial (i.e. $\operatorname{dim} M_{1} \geq 2, \operatorname{dim} M_{2} \geq 2$ ) foliations with $M_{1}, M_{2}$ compact. If there is a Lie algebra isomorphism $\Phi$ of $\mathcal{X}\left(M_{1}, F_{1}\right)$ onto $\mathcal{X}\left(M_{2}, F_{2}\right)$ then there is a foliation preserving diffeomorphism $\varphi$ of $M_{1}$ onto $M_{2}$ such that $\varphi_{*}=\Phi$.

For simplicity sake we restrict our attention in the proof to transversely orientable foliations.

Let us indicate analogous results to Theorems 1.1 and 1.2. The case of compact foliations was solved in [6] as well as the case of transversely complete foliations in [11]. Two very special cases of codimension one foliations were aiso considered in [6, Theorems $C$ and D]. In the last section of our note we formulate briefly a conjecture broadly generalizing these results.

In this note all manifolds are second countable and all manifolds and folia. tions are of class $C^{\infty}$. However, all considerations in the section 2 remain true in the case of $C^{2}$ foliations and $C^{2}$ functions.

Finally I would tike to thank Dr. Robert Wolak for fruitful conversations. I also express my deep gratitude to the referee whose critical remarks helped me to improve this note.

\section{Structure of open saturated sets}

Various authors have studied a theory of open saturated sets of one codimensional foliations. References for the facts presented in this section are Cantwell and Conlon [2], Hector [3] and Hector and Hirsch [4]. We shall formulate and prove here some preparatory results which will be useful in the sequel.

Let $(M, F)$ be a foliated compact manifold with codimension one. There is a increasing sequence $M_{k}, k=0,1,2, \ldots$, of closed saturated subsets of $M$ such that

1) $M_{0}$ is the union of all minimal sets of $F$;

2) if $M_{k-1}$ is defined for $k>0$ then the set $M_{k} \backslash M_{k-1}$ is the union of all minimal sets of $F \mid M \backslash M_{k-1}$.

Each leaf contained in $M_{k} \backslash M_{k-1}$ is called a leaf at level $k$. In general, a leaf contained in some $M_{k}$ is said to be at finite level. On the contrary, if there are any leaves in $M \backslash \bigcup\left\{M_{k}, k=0,1, \ldots\right\}$ they are said to be at infinite level. Let a leaf $L$ be at infinite level. The structure of $L$ is the union of all leaves of $\bar{L}$ that are at finite level. Then Theorem 5.0 in [2] states that the substructure of $L$ is dense in $\bar{L}$.

Our object of interest are local minimal sets. A local minimal set is a minimal set of $F \mid U$, where $U$ is some open saturated set. In particular, every proper leaf is a local minimal set. Each leaf of a local minimal set lies at certain finite level. On the other hand, leaves that lie in no local minimal set are at infinite level. 
We recall that minimal sets can be either closed leaves, or exceptional sets, or the whole manifold $M$. Denote by $C(F), E(F)$ and $Z(F)$ the union of all closed leaves, the union of all exceptional minimal sets and the union of all minimal sets of $(M, F)$ respectively. Let $U$ be any open saturated set. Then $Z(F \mid U) \neq \phi, C(F \mid U)$ is closed and $E(F \mid U)$ is a finite union (cf. [3]). In particular, the set $Z(F \mid U)$ is closed in $U$.

From now on we fix a foliation $T$ transversal to $F$; it is one dimensional and orientable. Following [3] let us consider a situation near a proper leaf $L$. Having fixed $T$ one can construct a pseudogroup $P$ of giobal holonomy on an orbit $C$ of $T$. Let $x \in C \cap L$. It is essential that there is a compact neighborhood $V$ of $x$ contained in a $T$-plaque with a family $f_{n}, n=1,2, \ldots$, of functions generating $P$ on $V$ and defined on $V$. We may identify $x$ with 0 and $V$ with an interval, say $[-1,1]$. The action of $P_{x}$, the isotropy subpseudogroup at $x$, induces on $V$ an equivalence relation. Shrinking $V$ if necessary, we have the following three types of minimal subsets of $[-1,1]$ : (i) a single point; (ii) a cyclic orbit i.e. a sequence $\left(x_{n}\right)$ in $(0,1]$ or $[-1,0)$ converging to 0 ; (iii) the intervals $(0,1]$ or $[-1,0)$.

Denotation. Let $\mathcal{U}$ be a union of all leaves which are not locally dense and have an open saturated neighborhood $U$ such that $F \mid U$ is without holonomy.

Then $\mathcal{U}$ is open and saturated. Of course, $\mathcal{U}$ need not contain all leaves without holonomy.

Lemma 2.1. Let $L$ be a proper leaf at level $k$ such that there is an open saturated neighborhood of $L$ consisting of proper leaves at level $k$ only. Then $L \subset \mathcal{U}$.

Proof: Let $V$ be a compact neighborhood of $x \in L$ contained in a $T$-plaque. We may assume that $V$ meets only proper leaves at level $k$. Hence the types (ii) and (iii) above are not admissible on $V$. Hence $F \mid \hat{V}$ is without holonomy, where $\hat{V}$ is the saturation of $V$.

As a consequence of Lemma 2.1 we have the following

Proposition 2.2. Let $L$ be a leaf at finite level such that $L \not \subset U$. Then there is an open saturated neighborhood $U$ of $L$ with the following property: for any $G \subset U$, an open saturated neighborhood of $L$, there exists $W$, an open saturated subset of $G$, such that $L \subset \dot{W}$ and any smooth function on $G$ constant along the leaves of $F \mid G$ is constant on each component of $W$.

Proof: The leaf $L$ may be proper, locally dense or exceptional. The proof is trivial if $L$ is locally dense. If $L$ is exceptional then $L$ is contained in an exceptional minimal set $N$. Then we apply Theorem 4.1 .1 in [4] which establishes the existence of an open saturated neighborhood $U$ of $N$ such that $N \subset \overline{L^{\prime}}$ for any $L^{\prime}$ in $U$. Therefore any smooth function constant along the leaves which is defined on some $W \subset U$, a neighborhood of $L$, is a constand function. 
Let us take up the case of $L$ proper at level $k$. Let $U$ be an open saturated set such that $L$ is minimal of $F \mid U$. We set $W=U \backslash C(F \mid U)$. Let $W^{\prime}$ be a connected component of $W$. Its boundary consists of a finite number of leaves $L_{1}, \ldots, L_{\mathrm{r}}$ at level $k$. Each leaf in $W^{\prime}$ spirals onto one of the leaves $L_{1}, \ldots, L_{r}$ or onto one of exceptional minimal sets $N_{r+1}, \ldots, N_{s}$ contained in $W^{\prime}$. Therefore a function on $U$ constant along the leaves of $F \mid U$ can assume only a finitc number of values on $W^{\prime}$ and thus it is constant on $W^{\prime}$. Finally, by Lemrna 2.1 and by the assumption, we get $L \subset \bar{W}$. Notice that in the above argument $U$ may be replaced by any open saturated $G \subset U$ such that $L \subset G$.

This completes the proof of the proposition.

H. Imanishi and $\mathrm{K}$. Yagi in [8] have studied a generalized Reeb component. We recall that a compact foliated manifold $(N, \mathcal{F})$, where $\partial N \neq \phi$, is called a generalized Reeb component if the following two conditions are satisfied: (1) all leaves in $\operatorname{Int}(N)$ are non-compact and proper; (2) $\mathcal{F} \mid \operatorname{Int}(N)$ has trivial holonomy.

There was shown the following

Proposition 2.3. (6. Proposition 2.1 in $[8]$ ). Let $(N, \mathcal{F})$ be a generalized Reeb component. Then there is a transverse vector field $X$ on $\operatorname{Int}(N)$ such that $X$ has an orbit $C$ and $C \cap L=\{$ one point $\}$ for any leaf $L$ in $\operatorname{Int}(N)$.

Such a vector field is said to be nice and $C$ is a nice orbit.

Let $U$ be an open saturated set. By $U^{c}$ we denote the completion of $U$ (cf. [2] or [4]). Let $i^{c}: U^{c} \rightarrow M$ be a canonical extension of the inclusion $i: U \hookrightarrow M$. Recall that $U^{c}$ is a manifold with boundary and $i^{c}$ maps diffeomorphically each component of $U^{c}$ onto a peripheral leaf of $U$.

Now if $U$ is a connected component of the set $U$ we have the following two possibilities:

(i) $U^{c} \cong L \times[0,1]$ is foliated as a product by the foliations $F$ and $T$;

(ii) $U^{c}$ is a manifold with boundary, there is a finite number of leaves which are the connected components of $\partial U^{c}$ and the restriction $F \mid \operatorname{In} t\left(U^{c}\right)$ is a fibraton over $S^{1}$.

Then we have the following corollary.

Corollary 2.4. There is a nice vector field on $\mathcal{U}$.

\section{Maximal ideals of $\mathcal{X}^{\prime \prime}(M, F)$}

By $\mathcal{X}^{\prime \prime}(M, F)$ we denote the derived ideal of $\mathcal{X}(M, F)$ i.e. the ideal generated by all brackets $[X, Y]$, where $X, Y \in \mathcal{X}(M, F)$.

We begin with the following proposition which is true for an arbitrary foliation $(N, \mathcal{F})$. 
Proposition 3.1. The derived ideal $\mathcal{Y}^{\prime}(N, \mathcal{F})$ coincides with $\mathcal{Y}(N, \mathcal{F})$.

The proof is a modification of that of Lemma 14 in [11] and is omitted.

Corollary 3.2. $\mathcal{Y}(N, \mathcal{F})$ is an ideal of $\mathcal{X}^{\prime \prime}(N, \mathcal{F})$.

Corollary 3.3. For any manifold $N$ the derived ideal $\mathcal{X}^{\prime \prime}(N)$ coincides with $\mathcal{X}(N)$.

Let $X \in \mathcal{X}(M, F)$. We have the unique decomposition $X=X_{1}+X_{2}$, where $X_{1}$ is tangent to $F$ and $X_{2}$ is tangent to the transversal foliation $T$. Before giving a description of maximal ideals we shall show that any $X \in \mathcal{X}^{\prime \prime}(M, \mathcal{F})$ is tangent to $F$ on $M \backslash \mathcal{U}$, that is $X_{2}=0$ on $M \backslash \mathcal{U}$.

Let $L \subset M \backslash U$ be an arbitrary leaf at finite level. If there does not exist any $X \in \mathcal{X}(M, F)$ such that $X$ is not tangent to $L$ then we are done. Suppose that $X \in \mathcal{X}(M, F)$ such that $X_{2} \neq 0$ on $L$. Hence $X_{2} \neq 0$ on an open saturated neighborhood $G$ of $L$. Let us consider any bracket $[Y, Z] \in \mathcal{X}^{\prime \prime}(M, F)$ such that $Y=Y_{1}+Y_{2}$ and $Z=Z_{1}+Z_{2}$ belong to $\mathcal{X}(M, F)$. Then there exist smooth functions $f$ and $g$ on $G$, constant along leaves, such that $Y_{2}=f X_{2}$ and $Z_{2}=g X_{2}$. By Proposition 2.2, there is an open saturated subset $W$ of $G$ such that $L \subset \bar{W}$ and $f, g$ are constant on each connected component of $W$. Hence

$$
\begin{aligned}
{[Y, Z] } & =\left[Y_{1}+Y_{2}, Z_{1}+Z_{2}\right] \\
& =\left[Y_{1}, Z_{1}\right]+\left[Y_{1}, Z_{2}\right]+\left[Y_{2}, Z_{1}\right]+\left[Y_{2}, Z_{2}\right] \\
& =\left[Y_{1}, Z_{1}\right]+g\left[Y_{1}, X_{2}\right]+f\left[X_{2}, Z_{1}\right]
\end{aligned}
$$

is tangent to $F$ on $W$ and, by continuity, also on $L$.

Next, let $L$ be at infinite level. The substructure of $L$ is obviously contained in $M \backslash \mathcal{U}$ and by the above argument $\mathcal{X}^{\prime}(M, F)$ is tangent to $F$ on the substructure. Hence, by continuity, any element of $\mathcal{X}^{\prime}(M, F)$ is tangent to $L$.

Thus we have the proof of the following

Theorem 3.4. Let $(M, F)$ be an arbitrary one codimensional foliation on a compact manifold $M$. There is an open saturated subset $U$ of $M$ such that $F \mid \mathcal{U}$ is without holonomy and any $X \in \mathcal{X}^{\prime}(M, F)$ is tangent to $F$ on $M \backslash \mathcal{U}$.

Remark. It is known that any one codimensional foliation without holonomy admits an extension of any vector at a point to a foliated vector field. Our theorem completes this property: the transversal part of $\mathcal{X}^{\prime}(M, F)$ simply vanishes outside an open set without holonomy.

Let $C$ be a nice orbit on $\mathcal{U}$ of $T$ (now we consider $T$ on $\mathcal{U}$ only). 
Corollary 3.5. The transversal part $X_{2}$ of $X \in \mathcal{X}^{\prime}(M, F)$ is completely determined by its restriction $X_{2} \mid C$.

In fact, the flow of $X_{2} \mid C$ uniquely determines the flow of $X_{2}$.

Deftnition. From now on, by $\operatorname{Trans}(M)$ we denote the union of all leaves $L$ such that there is $X \in \mathcal{X}^{\prime}(M, F)$ with $X_{2} \neq 0$ on $L$.

Trans $(M)$ is open, saturated and contained in $\mathcal{U}$ by Theorem 3.4. The following examples justify the definition.

Examples: (3.6) Let $M=T^{2}$ and $F$ be the well known foliation by a rotation with rational slope. Here the transversal part of $\mathcal{X}(M, F)$ is isomorphic to $\mathcal{X}\left(S^{1}\right)$. Since, by Corollary $3.3, \mathcal{X}^{\prime}\left(S^{1}\right)=\mathcal{X}\left(S^{1}\right)$ we have $\mathcal{X}^{\prime}(M, F)=$ $\mathcal{X}(M, F)$. In particular, $\operatorname{Trans}(M)=M$.

(3.7) The situation is quite different in the case of the Reeb foliation of $S^{3}$. Obviously $U=S^{3} \backslash T^{2}$, where $T^{2}$ denotes the unique compact leaf. A nice orbit consists of two disjoint circles. However, the transversal part of $\mathcal{X}(M, F)$ is finite dimensional as it was shown by $\mathrm{K}$. Fukui in [5]. Consequently $\operatorname{Trans}(M)=\phi$.

Now we shall try to describe maximal ideals. Let $N$ be a manifold and $p \in N$. By $m_{p}$ we denote the ideal of $\mathcal{X}(N)$ formed by all vector fields vanishing at $p$ with all its derivatives. By abuse of notation, $m_{p}$ denotes also such an ideal in any subalgebra of $\mathcal{X}(N)$. The following fact is fundamental.

Proposition 3.8. Let $(N, \mathcal{F})$ be any compact foliated manifold and $\operatorname{dim} \mathcal{F}>$ 0 . The ideals $m_{p}, p \in N$, are the unique maximal ideals in $\mathcal{Y}(N, \mathcal{F})$. In partic. ular, $m_{p}$ are the unique maximal ideals in $\mathcal{X}(N)$.

The second part was proved in [10]. The proof of the first part is essentially the same.

Denotation. For any algebra $A$ we denote by $A^{*}$ the set of all maximal ideals of $A$.

For simplicity sake we shall write $\mathcal{A}$ instead of $\mathcal{X}^{\prime}(M, F)$.

One can easily see that $m_{p} \in \mathcal{A}^{*}$ for $p \in M \backslash \operatorname{Trans}(M)$. Let $L$ be a leaf in Trans $(M)$. Then $\mu_{L}$ denotes the ideal in $\mathcal{A}$ of all vector fields with their transversal parts infinitely fiat on $L$. A standard argument shows that $\mu_{L} \in \mathcal{A}^{*}$.

Lemma 3.9. For any $p \in \operatorname{Trans}(M)$ the ideal $m_{p}$ is contained in $\mu_{L}$, where $p \in L$.

In fact, if the fiow of a foliated vector field fixes $p$ then it fixes, of course, the leaf $L$.

Now let us consider the Lie algebra $\mathcal{A}$ restricted to $\operatorname{Trans}(M)$. By the above arguments, the transversal part of $\mathcal{A}$ may be identified with a subaigebra, say $A$, of $\mathcal{X}(\mathcal{C})$, where $\mathcal{C}$ is a topological sum of a number of $S^{1}$ or $(0,1)$. In particular, 
the ideals $\mu_{L}$, where $L \subset \operatorname{Trans}(M)$, correspond to $m_{x}, x \in \mathcal{C}$. On the other hand, there are some maximal ideals in $A$ different from $m_{x}$. Let $\left\{\lambda_{i}\right\}$ be the totallity of such ideals. For any ideal $\lambda_{i}$ there is the unique corresponding ideal $\bar{\lambda}_{i} \in \mathcal{A}^{*}$. Suck ideals exist, even for very simple foliations, as the following example shows.

Example 3.10. Let a sequence $\left(x_{k}\right) \subset(0,1), k=1,2, \ldots$, converge to 0 . By $\lambda\left(x_{k}\right)$ we denote the ideal of all $X \in \mathcal{X}((0,1))$ such that $X$ is $\alpha_{k}$-flat at $x_{k}$ for some sequence of positive integers $\alpha_{k}$ tending to $\infty$. A maximal ideal containing $\lambda\left(x_{k}\right)$ gives an example of such $\lambda_{i}$.

Proposition 3.11. The unique elements of $\mathcal{A}^{*}$ are $m_{p}, p \in M \backslash$ Trans $(M), \mu_{L}, L \subset \operatorname{Trans}(M)$, and $\vec{\lambda}_{i}$.

Proof: Let $I$ be a maximal ideal of $\mathcal{A}$ such that $I$ is neither contained in $m_{p}$, nor in $\mu_{L}$, nor in $\bar{\lambda}_{i}$. Let $I^{\prime}=I \cap \mathcal{Y}(M, F)$. Then, by Proposition 3.8 and Lemma 3.9 , we get $I^{\prime}=\mathcal{Y}(M, f)$ and $\mathcal{Y}(M, F) \subset I$. Therefore there is a unique $J$, an ideal of $A$, corresponding to $I$. Then $J$ being proper must be contained in $m_{x}$ or $\lambda_{i}$. Hence $I$ is contained in $\mu_{L}$ or $\bar{\lambda}_{i}$. This contradiction proves the proposition.

Definition. Maximal ideals $\mu_{L}$ and $\bar{\lambda}_{i}$ are called "horizontal"; $m_{p}$ are called "vertical". We shall denote

$$
\begin{aligned}
& \Sigma=\text { the totallity of } \mu_{L}, \\
& \Xi=\text { the totallity of } m_{p}, \\
& \Lambda=\text { the totallity of } \bar{\lambda}_{i} .
\end{aligned}
$$

\section{Stone topology of $\mathcal{A}^{*}$ and the proof of Theorem 1.2}

In the situation described above we have to find an algebraic property which characterizes the horizontal ideals. This property being preserved by isomorphisms ensures that $\mu_{L}$ cannot be mapped onto $m_{p}$ and vice versa. Our characterization is based on topological properties of $\mathcal{A}^{*}$.

We introduce the Stone topology on the set $\mathcal{A}^{*}$ in the following way. Let $\Omega$ be a subset of $\mathcal{A}^{*}$. Then the closure of $\Omega$ is defined by

$$
\bar{\Omega}=\left\{\mu \in \mathcal{A}^{*}: \bigcap\{w \in \Omega\} \subset \mu\right\} .
$$

In particular, $\bar{\phi}=\phi$.

Remark, Let $(N, \mathcal{F})$ be any foliated manifold with $\operatorname{dim} \mathcal{F}>0$. It is an easy consequence from Proposition 3.8 that the mapping $N \ni p \rightarrow m_{p} \in \mathcal{Y}(N, \mathcal{F})^{*}$ is a homeomorphism. Specifically, $N$ and $\mathcal{X}(N)^{*}$ are homeomorphic.

Now we consider the partition of $\mathcal{A}^{*}$ by connected components in the Stone topology. We want to show that such a component must be "vertical" i.e. formed by vertical ideals, or "horizontal" i.e. formed by horizontal ideals.

First we strengthen Theorem 3.4 . 
Theorem 4.1. The transversal part $X$ of a vector field belonging to $\mathcal{A}$ vanishes with all its derivatives on $M \backslash \operatorname{Trans}(M)$.

Proof: Let $p \in L$ and $L \not \subset \operatorname{Trans}(M)$.

First case: $L \subset \mathcal{U}$. There is a foliated vector field $Y$ on $M$ tangent to $T, Y(p) \neq 0$. Let $\left(U, x_{1}, \ldots, x_{n}\right)$ be a bidistinguished chart at $p$ (with respect to $F$ and $T$ ) such that $Y=\partial / \partial x_{n}$ near $p$. Suppose that there is $k>0$ such that the $\mathrm{k}$-th derivative $\partial^{k} X / \partial x_{n}^{k} \neq 0$ at $p$. We have $[Y, X] \in \mathcal{X}(M, F)$ and $[Y, X]=\partial X / \partial x_{n}$ on a neighborhood of $p$. Moreover, $X \in \mathcal{A}$ implies $[Y, X] \in \mathcal{A}$. Continuing this procedure $k$ times we get $X_{0} \in \mathcal{A}$ such that $X_{0}(p) \neq 0$. This contradicts the definition of $\operatorname{Trans}(M)$.

Second case: $L \not \subset \mathcal{U}$. Since Trans $(M) \subset \mathcal{U}$ is an open saturated set without holonomy one can construct a non-vanishing foliated vector field $Y$ on Trans $(M)$ tangent to $T \mid \operatorname{Trans}(M)$. Now, if $X=f Y$ on $\operatorname{Trans}(M)$ then $f$ is a smooth function on $\operatorname{Trans}(M)$ constant along leaves.

First we assume $L$ to be at finite level. If $L$ is locally dense or exceptional then $X=0$ on a neighborhood of $p$ (see the proof of Prop. 2.2). Next, let $L$ be proper and $U$ be an open saturated neighborhood of $L$ such that $L$ is a minimal set of $U$. Then $W=U \backslash C(F \mid U)$ satisfies $L \subset \bar{W}$. Let $L \not \subset \overline{W \cap \operatorname{Trans}(M)}$. There is $G$, an open neighborhood of $L$, such that $G \cap W \cap$ Trans $(M)=\phi$ and $G \cap W \neq \phi$ since $L \not \subset \mathcal{U}$. Then $X=0$ on $G \cap W, \partial X=0$ on $G \cap W$ and so on. Thus $X$ is infinitely fiat on $G \cap W$ and at $p$. Next, let $L \subset W \cap \operatorname{Trans}(M)$. As in the proof of 2.2 , the function $f$ is constant on each connected component of $W \cap \operatorname{Trans}(M)$. Therefore $f^{\prime}=\partial f=0$ on $W \cap \operatorname{Trans}(M)$ and $\partial X=0$ on $W \cap \operatorname{Trans}(M)$ and so on. In fact, locally $Y=\partial / \partial x_{n}$ in some distinguished chart. Hence, by continuity, $X$ is infinitely flat at $p$.

Finally, let us consider $L$ at infinite level. It is visible that for any $L_{\alpha}$ of the substructure of $L$ there is an open set $W_{\alpha}$ such that $X$ is infinitely flat on $W_{\alpha}$ and $L_{\alpha} \subset \bar{W}_{\alpha}$. Hence $X$ is infinitely flat on the union $W=\bigcup W_{\alpha}$ and $L \subset \overline{U L_{\alpha}} \subset \bar{W}$. Thus $X$ is infinitely flat at $p$.

This completes the proof.

Corollary 4.2. We have $\bar{\Sigma} \cap \bar{\Xi}=\phi$ and $\bar{\Lambda} \cap \bar{\Xi}=\phi$. In particular, the components of $\mathcal{A}^{*}$ are purely "vertical" or purely "horizontal".

Proof: $\overline{\Sigma U \Lambda}=\Sigma \cup \Lambda$ follows from the evident property that the vanishing with derivatives of transversal parts of foliated vector fields cannot yield the vanishing of their tangent parts. On the other hand, the above theorem implies $\bar{\Xi}=\Xi$. In fact, let $\lambda$ be any horizontal ideal. If $X$ is a transversal of an element of $A$ which does not belong to $\lambda$ then, by the theorem, $X \in \bigcap \equiv$. Then the definition of the topology implies that $\lambda \notin \vec{E}$.

Definition. $\mu \in \mathcal{A}^{*}$ is said to be distinguished if $\mu$ belongs to an element of a unique minimal family $R$ of connected components of $\mathcal{A}^{*}$ characterized by 
the equality

$$
\bigcap\left\{\mu \in(\bigcap \tilde{R})^{*}\right\}=0
$$

where $\tilde{R}=\bigcup R$.

Lemma 4.3. There does not exist any connected component of $\mathcal{A}^{*}$ consisting of elements of $\Lambda$ only.

In fact, it is immediate from the definition that $\bar{\Sigma}=\Sigma \cup \Lambda$.

Proposition 4.4. The horizontal ideals are uniguely characterized by the property "to be distinguished".

Proof: In fact, the intersection of all horizontal ideals gives $\mathcal{Y}(M, F)$. It is clear that $\mathcal{Y}(M, F)^{*}$ satisfies the definition by Proposition 3.8 .

Suppose now that $R$ does not contain some horizontal component, say $\Sigma^{\prime}$. By Lemma 4.3, there are a connected component $U$ of $\operatorname{Trans}(M)$ and a leaf $L \subset U$ such that $\mu_{L} \notin \tilde{R}=\bigcup R$. Now, it follows from the definition of the topology that $\mu_{L^{\prime}} \in \Sigma^{\prime}$ for every $L^{\prime} \subset U$. Consequently, $\bigcap\left\{\mu \in(\cap \tilde{R})^{*}\right\}$ contains all vector fields tangent to $F \mid U$ with supports in $U$ and thus it is non-zero. This and the minimality of $R$ implies the proposition.

Corollary 4.5. $\mathcal{Y}(M, F)$ is characterized in $\mathcal{A}$ as the intersection of all distinguished ideals.

Now we have to reformulate slightly Theorem 1.1 .

Corollary 4.6. If $\Phi$ is a Lie algebra isomorphism of $\mathcal{X}\left(M_{1}, F_{1}\right)$ onto $\mathcal{X}\left(M_{2}\right.$, $\left.F_{2}\right)$ such that $\Phi\left(\mathcal{Y}\left(M_{1}, F_{1}\right)\right)=\mathcal{Y}\left(M_{2}, F_{2}\right)$, then there is a foliation preserving diffeomorphism $\varphi$ of $M_{1}$ onto $M_{2}$ such that $\varphi_{*}=\Phi$ on $\mathcal{X}\left(M_{1}, F_{1}\right)$.

The proof is in [11].

The proof of Theorem 1.2 is now a consequence of Corollaries 4.5 and 4.6 and of the fact that an isomorphism of Lie algebras induces an isomorphism of their derived ideals.

Remark. Theorem 1.2 can be formulate in slightly more general version. Namely, $\mathcal{X}\left(M_{1}, F_{1}\right)$ and $\mathcal{X}\left(M_{2}, F_{2}\right)$ can be replaced by any their subalgebras $\mathcal{A}_{1}$ and $\mathcal{A}_{2}$ respectively such that $\mathcal{Y}\left(M_{1}, F_{1}\right) \subset \mathcal{A}_{1}$ and $\mathcal{Y}\left(M_{2}, F_{2}\right) \subset \mathcal{A}_{2}$. The proof is the same.

\section{Conjecture}

A. Lichnerowicz introduced a notion of Jacobi manifold (see e.g. [7]) which correponds to a notion of local Lie algebra over $R$. In particular, this notion 
is a generalization of symplectic manifold as well as of contact manifold. If a Jacobi manifold is non-transitive, it determines a generalized foliation. Of course, an infinitesimal automorphism of Jacobi structure is a foliated vector field of the corresponding generalized foliation.

Now our conjecture is the following: the Lie algebra of infinitesimal automorphisms of Jacobi manifold determines completely the underlying smooth structure of manifold and the Jacobi structure itself. Notice that Pursell-Shanks type theorems proved by $\mathrm{H}$. Omori in $[9$, chapter $\mathrm{X}]$ are a partial solution of this conjecture in the case of transitive Jacobi manifold. The reduction of the case non-transitive to the case transitive is, in fact, a generalization of Theorem 1.2 .

An interpretation of the conjecture in the hamiltonian mechanics is the following. One can consider a Jacobi manifold as a generalized phase-space and the Lie algebra of infinitesimal automorphisms as the algebra of symmetries of this phase-space. Then the conjecture states that the structure of phase-space is uniquely determined by the algebra of its symmetries.

\section{References}

1. I. AmemiYa, Lie algebra of vector fields and complex structure, $J$. Math. Soc. Japan 27 (1975), p. 545.

2. J. CANTWELl, L. Conlon, Poincaré-Bendixson theory for leaves of codimension one, Trans. Amer. Math. Soc. 265, 1 (1981), p. 181.

3. G. HeCTOR, Architecture des feuilletages de classe $C^{2}$, Asterisque 107-108 (1983), p. 245.

4. G. HECTOR, U. HIRSCH, "Introduction to the Geometry of foliations," Part B, Vieweg and Sohn, 1983.

5. K. FUKUI, On the homotopy type of some subgroups of Diff $M^{3}$, Japan J. Math. 2, 2 (1976), p. 249.

6. K. FUKUI, N. TOMITA, Lie algebra of foliation preserving vector fields, $J$. Math. Kyoto Univ. 22, 4 (1983), p. 685.

7. F. Guedira, A. Lichnerowicz, Géométrie des algèbres de Lie locales de Kirillov, J. Math. pures et appl. 63 (1984), p. 407.

8. H. IMANISHI, K. YAGI, On Reeb components, J. Math. Kyoto Univ. (1976).

9. H. OMORI, "Infinite dimensional Lie transformation groups," Lecture Notes 427, Springer-Verlag, 1976. 
10. L.E. Pursell, M.E. Shanks, The Lie algebra of smooth manifold, Proc. Amer, Math. Soc. 5 (1954), p. 468.

11. T. RyBicki, On the Lie algebra of a transversally complete foliation, $P u b$. Mat. UAB 31, 1 (1987), p. 5.

12. R. WolaK, Maximal ideals in $\operatorname{Loc}(M, F)$, Collect. Math. 38 (1987), p. 157.

\author{
Instytut Matematyki \\ Uniwersytet Jagiellonski \\ ul.Reymonta 4 \\ 30-059 Krakow \\ POLAND
}

Rebut el 21 de Novembre de 1989 